\title{
Rapid Detection of Norovirus Using Paper-based
}

\author{
Xuan Weng ${ }^{1}$, Suresh Neethirajan ${ }^{1} *$
}

$21 *$ Correspondence: sneethir@uoguelph.ca (S. Neethirajan)

22 BioNano Laboratory, School of Engineering, University of Guelph, Guelph, Canada 


\section{Abstract}

24 Noroviruses (NoV) are the leading cause of outbreak of acute gastroenteritis worldwide. A

25 substantial effort has been made in the development of analytical devices for rapid and sensitive

26 food safety monitoring via the detection of foodborne bacteria, viruses and parasites.

27 Conventional analytical approaches for noroviruses suffer from some critical weaknesses: labor-

28 intensive, time-consuming, and relatively low sensitivity. In this study, we developed a rapid

29 and highly sensitive biosensor towards point-of-care device for noroviruses based on 6-

30 carboxyfluorescein (6-FAM) labeled aptamer and nanomaterials, multi-walled carbon nanotubes

31 (MWCNTs) and graphene oxide (GO). In an assay, the fluorescence of 6-FAM labeled aptamer

32 was quenched by MWCNTs or GO via fluorescence resonance energy transfer (FRET). In the

33 presence of norovirus, the fluorescence would be recovered due to the release of the 6-FAM

34 labeled aptamer from MWCNTs or GO. An easy-to-make paper-based microfluidic platform

35 made by nitrocellulose membrane was used to conduct the assay. The quantitative detection

36 of norovirus virus-like particles (NoV VLPs) was successfully performed. A linear range of 0-

$37 \quad 12.9 \mu \mathrm{g} / \mathrm{mL}$ with a detection limit of $40 \mathrm{pM}$ and $30 \mathrm{pM}$ was achieved for the MWCNTs and GO

38 based paper sensors, respectively. The results suggested the developed paper-based microfluidic

39 device is simple, cost-effective and holds the potential of rapid in situ visual determination for

40 noroviruses with remarkable sensitivity and specificity, which provides a new way for early

41 identification of NoV and thereby an early intervention for preventing the spread of an outbreak.

42 Keywords: Norovirus; Paper-based microfluidics; Nitrocellulose membrane; Multi-walled

43 carbon nanotubes; Biosensor 


\section{Introduction}

47 Noroviruses (NoV) are the leading causes of acute viral gastroenteritis [1], as well as one of the

48 leading causes of foodborne disease outbreaks and the most common cause of infectious

49 gastroenteritis worldwide [2], clinical symptoms including abdominal pain, diarrhea, muscle

50 ache, and mild fever [3]. NoV infection may be direct from person to person or transmitted

51 through food and drinks (ready-to-eat foods, table water) and the outbreak of which is frequently

52 occurred in community facilities such as hospitals and school cafeterias. Currently, NoV are

53 usually detected after an outbreak on the suspected food with a range of methods, including

54 enzyme-linked immu- nosorbent assay (ELISA) [4], western blot [5], and nucleic acid-based

55 methods, such as reverse transcription polymerase chain reaction (RT-PCR) [6] etc. These

56 methods usually require expensive reagents, specialized equipment, and skillful researchers to

57 perform the analyses and long waiting time for the results. In addition, antibodies are usually

58 used in the biomolecular recognition, however, antibodies are usually expensive, have limited

59 shelf-life and false-positive issues may occur due to the cross-reactivity between the antibodies

60 and non-target molecules. Since the NoV have high resistance to routine disinfection and low

61 infection dose, early on-site detection is extremely important for the prevention of the

62 transmission and effective controlling of the infection as well as the spread of an outbreak. In

63 addition, NoV, in cases, can be pre-emptively identified to efficiently block and reduce the

64 infection [7]. FDA of United States approved the first early detection NoV test in 2011. All of

65 the aforementioned reasons urge the need for more rapid, accurate, and ultra-sensitive assays to

66 detect potential NoV contamination in complex food matrices. Development of such a method

67 for NoV associated with foodborne illness can be a powerful tool for the application in response

68 to outbreak management. 
To date, researchers have put numerous efforts in the development of rapid and efficient

70 methods, and biosensor is one of the promising ways to provide alternative to conventional

71 assays due to its features of rapid, simple, reproducible, and cost-effective. Hong et al. [8]

72 reported an electrochemical biosensor for pre-emptive on-site NoV detection by using

73 nanostructured gold electrode conjugated with a NoV capturing agent concanavalin A. The

74 biosensor was able to detect $\mathrm{NoV}$ in a concentration range of $10^{2}$ and $10^{6}$ copies/mL within 1

75 hour. Hwang et al. [9] reported a label-free electrochemical biosensor for human NoV detection

76 using affinity peptide and a limit of detection (LoD) of 7.8 copies/mL was achieved. Although

77 electrochemical biosensors are able to provide high sensitivity detection, the relative high price

78 make them not suitable to be used in disposable chips for on-site detection [10]. Lateral-flow

79 assays (LFAs) have been well developed and widely used in point-of-care testing (POCT), which

80 have also been applied for virus detection $[11,12]$. The main drawbacks of most of LFAs are

81 the low sensitivity and the yes/no results. The introduction of nanomaterial contributes to the

82 enhancement of sensitivity. Hagström et al. [13] developed a LFA for the detection of NoV by

83 using nanoparticle reporters and a LoD of $10^{7}$ virus-like particles per microliter was obtained.

84 Paper-based microfluidic devices, as alternative to the traditional microfluidic devices made

85 by PDMS, glass, silicon or other polymers, find their applications in biochemical, health

86 diagnosis and food safety fields due to its unique advantages, including the low cost, easy-to-

87 fabricate process and the simplicity $[14,15]$. In addition, paper-based microfluidic devices are

88 powerless due to the capillary action of the hydrophobic channel. Currently, wax printing

89 technique is being used to fabricate the paper-based microfluidic devices.

90 Therefore, we developed a simple, low-cost and easy-to-make paper-based microfluidic

91 device with multi-detection capability for determination of virus contaminants in food. NoV was 
92 used as the testing model. Carbon nanostructures usually have a wide range of absorption

93 spectrum overlapping with the fluorescence spectra of various fluorophores [16], which allows

94 FRET between them. The paper-based microfluidic device utilized FRET between nanomaterials

95 and the virus probe functionalized fluorophore to quantitatively determine the concentration of

96 the sample. In the present work, 6-carboxyfluorescein (6-FAM) labeled norovirus aptamer

97 associated with MWCNTs or GO was employed as the "probe" to sensitively sense the presence

98 of NoV with high specificity. MWCNTs consist of multiple rolled layers (concentric tubes) of

99 graphitic sheets [17] while GO is a single-atom-thick two-dimensional carbon nanomaterial and

100 both of them are efficient quenchers to various fluorophores [18, 19]. In our study, the efficiency

101 of two kinds of nanomaterials namely, MWCNTs and GO, as the fluorescence quencher were

102 investigated. In addition, the sensing probe was operated on an easy-to-make paper-based

103 microfluidic device which is inexpensive and can be easily fabricated by a paper puncher within

104 a minute. Such paper-based microfluidic device is power free because sample can be driven by

105 the capillary force, and can be easily made within $1 \mathrm{~min}$. The design of the paper-based

106 microfluidic device allows for multiplex detection (up to 6). Another advantage of using paper

107 substrate is the avoidance of complicated processes of probe immobilization and surface

108 modification which can be achieved through the physical absorption. Schematic illustration in

109 the Fig. 1a and 1b shows the main principle of this sensor, as an example, only the MWCNTs is

110 illustrated. The principle of the sensing is based on the FRET between the fluorophores and

111 nanostructure quenchers. The conformational change of the aptamer associated with the distance

112 changes between the fluorophores and quenchers leads to a measurable fluorescence intensity. A

113 ready-to-use device was made by loading the complex "probe" consisting of nanomaterial and

114 target specific aptamer with fluorescence labelling onto the reaction wells and dries out. In an 
115 assay, sample was loaded onto the sample well followed by diffusion to the reaction wells

116 immediately and bind with the aptamers, leading to the recovery of the fluorescence. The

117 recovered fluorescence intensity was then used to quantitatively determine the target

118 concentration in the sample.

\section{Experimental}

\section{$121 \quad$ Materials and chemicals}

122 Carboxylic acid functionalized MWCNTs, graphene oxide, nitrocellulose membranes

123 (Whatman ${ }^{\circledR}$ Protran ${ }^{\circledR}$ ) and all other mentioned chemicals and solvents were purchased from

124 Sigma-Aldrich (Oakville, ON, Canada). The 6-carboxyfluorescein (6-FAM) binding norovirus

125 aptamer was synthesized by Integrated DNA Technologies, Inc. (Coralville, IA, USA) with the 126 sequence of 5'-AGT ATA CGT ATT ACC TGC AGC CCA TGT TTT GTA GGT GTA ATA

127 GGT CAT GTT AGG GTT TCT GCG ATA TCT CGG AGA TCT TGC-3’ [20]. The virus like

128 particles (VLPs) formed by group 2 Norovirus capsid antigen were purchased from 129 MyBioSource, Inc. (San Diego, CA, USA). The craft paper punch was purchased from 130 Amazon.ca (McGill@, Paper Blossoms Lever Punch-Multi Daisy, .625" To 1"). Unless

131 otherwise noted, all reagents were of analytical grade unless otherwise stated, all solutions were 132 prepared with double-distilled water.

\section{Preparation of 6-FAM Aptamer Functionalized and MWCNTs /GO}

136 The probe of 6-FAM aptamer was prepared by firstly resuspending the dried aptamer pellet in 137 the TE buffer (10 mM Tris $\mathrm{HCl}, 0.1 \mathrm{mM}$ EDTA, pH 8.0) and followed by incubation for $30 \mathrm{~min}$ 
138 at room temperature. Then a series of aptamer solution was made with folding buffer (1 mM

$\left.139 \mathrm{MgCl}_{2}, 1 \times \mathrm{PBS}, \mathrm{pH} 7.4\right)$ and heated at $85^{\circ} \mathrm{C}$ for $5 \mathrm{~min}$. This was then followed by cooling down

140 the dilutions before use.

141 MWCNTs and GO were diluted with DI water to a series of concentrations ranging from

1420.005 to $0.1 \mathrm{mg} / \mathrm{mL}$. Then we mixed the MWCNTs or GO dilutions with 6-FAM aptamer

143 working solutions of specific concentrations and incubated for a period of time to quench the

144 fluorescence of the aptamer. The mixture was then pipetted onto the detection area of the paper-

145 based microfluidic device. The incubation time was optimized by investigating the fluorescence

146 signal at time points of $1 \mathrm{~min}, 5 \mathrm{~min}, 10 \mathrm{~min}$ and $20 \mathrm{~min}$. Serial dilutions of NoV VLPs stocks

147 (10-fold) were prepared on a range of $0 \sim 12.9 \mu \mathrm{g} / \mathrm{mL}$ using $1 \mathrm{X}$ phosphate buffered saline (PBS

148 Buffer) solution.

\section{Paper-based microfluidic device fabrication and assay procedure}

151 The schematic of the paper-based microfluidic device design illustrating the sensing mechanism

152 as well as the picture of the device is shown in Fig. 1c and 1d. A blossoms lever-shaped paper

153 device was patterned manually as the paper-based microfluidic device on the nitrocellulose

154 membranes by a craft punch under room temperature. The width of the "arm" channels was 500

$155 \mu \mathrm{m}$, the diameters of the center area (sample loading point) and the detection area were $3 \mathrm{~mm}$

156 and $1 \mathrm{~mm}$, respectively.

157 Nitrocellulose membranes were used to make the paper-based microfluidic device.

158 Nitrocellulose membranes are a popular matrix that can be used for simple and rapid protein

159 immobilization because of its non-specific affinity for amino acids [21]. The mixture of 6-FAM

160 aptamer solution and MWCNTs or GO was pipetted onto the detection area of the paper-based 
161 microfluidic device and allowed to dry in the air for $20 \mathrm{~min}$. The dried paper was then undergone

162 the blocking treatment by using PBS with 5\% BSA and 0.05\% Tween-20 for 30 min. The

163 blocked paper device was then ready to use and could be stored at $4{ }^{\circ} \mathrm{C}$ for short term storage. It

164 is noted that the nitrocellulose membrane based paper device should be kept in a dry atmosphere

165 and away from noxious fumes, avoiding exposure to sunlight, which is conducive to extending

166 the shelf life of nitrocellulose membranes-based diagnostic device and maintaining the molecular

167 recognition capability of immobilized proteins for a long time, even a few years.

169 During an assay, the fluorescence intensities of the reaction (sensing) zone were recorded as

170 reference. Then aliquots of varying concentrations of NoV VLPs in PBS buffer (10 $\mu \mathrm{L})$ and

171 controls were loaded onto the central, the liquid would diffuse into the detection area

172 spontaneously due to the capillary force. After incubation, the fluorescence intensities of the

173 reaction (sensing) zone were measured again to quantitatively analyse the sample concentration.

174 Each sample was tested 3 times. Three independent experiments were carried out for each 175 conditions.

\section{Characterization and optimization}

177 Transmission electron micrographs were obtained using the FEI-Tecani G2, operating at $200 \mathrm{kV}$.

178 The fluorescent imaging $(\mathrm{Ex} / \mathrm{Em}=490 \mathrm{~nm} / 520 \mathrm{~nm})$ of the detection area was taken on a 179 fluorescent microscopy (Nikon Eclipse Ti, Nikon Canada Inc., Mississauga, ON, Canada). All 180 images were taken under the same settings, namely exposure time, magnification, etc. The 181 fluorescence intensity was then analyzed by Nikon NIS Elements BR version 4.13 software to 
182 investigate the concentration of the sample. For the validation tests, the fluorescence spectra

183 were recorded by the Cytation 5 Multi-mode Reader (BioTek, Winooski, VT, USA).

184 The ratio of the 6-FAM aptamer solution and MWNCTs/ GO, quenching and recovery time

185 were optimized. In a typical optimization test, $20 \mu \mathrm{L}$ of 6-FAM aptamer, $20 \mu \mathrm{L}$ of MWCNTs/

186 GO solution were well mixed. Fluorescence intensity was measured at the time points of $0,5 \mathrm{~min}$,

$18710 \mathrm{~min}, 15 \mathrm{~min}$ and $20 \mathrm{~min}$. Afterwards, $20 \mu \mathrm{L}$ of NoV VLPs standard solution was added and

188 mixed well. After incubation for a period time, the recovered fluorescence intensity of the

189 resulting solutions was analyzed. The relationship between the fluorescence intensity change and

190 the corresponding concentration was plotted. The fluorescence spectra were measured at Ex/

$191 \mathrm{Em}=490 \mathrm{~nm} / 520 \mathrm{~nm}$ by the Multi-mode Reader. All samples were prepared in triplicates.

193 Results and discussion

\section{Characterization, validation and optimization of the biosensor}

195 The TEM images of the carboxylic acid functionalized MWCNTs and GO clearly showed the

196 tubular and sheet nanostructures (Fig. 2a and 2b). The UV-Vis absorption spectra of the

197 carboxylic acid functionalized MWCNTs and bare GO are shown in Fig. 2c and as shown the

198 maximum absorption peaks appear at $253 \mathrm{~nm}$ and $258 \mathrm{~nm}$, respectively. Fig. 2d shows the

199 fluorescence spectra of the 6-FAM NoV aptamer. The fluorescence intensity peak is located at a

200 wavelength of $520 \mathrm{~nm}$.

201 The quenching effect of two nanomaterials, MWCNTs and GO, were investigated and

202 compared. In order to obtain optimum performance, the concentrations of the MWCNTs, GO,

203 NoV aptamer as well as the reaction (incubation) time were studied, respectively. For the 204 nanomaterials MWCNTs and GO, a series concentrations, 0.05, 0.1, 0.5 and $1.0 \mathrm{mg} / \mathrm{mL}$, were 
205 investigated, respectively. And for the NoV aptamer, $1 \mu \mathrm{M}$ and $5 \mu \mathrm{M}$ were chosen for further

206 experiments. The influence of reaction time on the fluorescence intensities of before and after

207 quenching were also studied. Fig. 3 and Fig. 4 show the fluorescence quenching and recovery of

208 the NoV aptamer of $1 \mu \mathrm{M}$ and $5 \mu \mathrm{M}$ by various of concentrations of MWCNTs and GO when

209 detecting the NoV sample of $129 \mathrm{ng} / \mathrm{mL}$. The validation of these optimization testing was

210 conducted by a microplate reader. All assays were performed at room temperature. As shown in

211 Fig. 3a and 3b, higher quenching effect was found at the $0.1 \mathrm{mg} / \mathrm{mL}$ of MWCNTs when NoV

212 aptamer was at $1 \mu \mathrm{M}$ while $0.05 \mathrm{mg} / \mathrm{mL}$ of MWCNTs had higher one when the concentration of

213 NoV aptamer increased to $5 \mu \mathrm{M}$. For the nanostructure $\mathrm{GO}$, there is no notable difference

214 between $0.05 \mathrm{mg} / \mathrm{mL}$ and $0.1 \mathrm{mg} / \mathrm{mL}$ was observed when NoV aptamer was at $1 \mu \mathrm{M}$ and $5 \mu \mathrm{M}$,

215 as shown in Fig. 4a and 4b. A slightly strong quenching effect was found by GO compared to

216 that of MWCNTs, which can be attributed to the more interaction area of the sheet structure of

217 GO than the tube structure of MWCNTs. Fig. 3c, 3d and Fig. 4c, 4d shows the recovering of the

218 fluorescence quenched by MWCNTs and GO, respectively. Remarkable recovery of the

219 fluorescence quenched appeared for both of them, which was attributed to FRET generated by

220 the synergy of particles collision and $\pi-\pi$ stacking interaction between them and aptamer [22].

221 However, the recovery level varies. For the MWCNTs, the optimum recovery appeared at the

222 setting of NoV aptamer of $5 \mu \mathrm{M}$ and MWCNTs of $0.1 \mathrm{mg} / \mathrm{mL}$, as shown in Fig.3d. While for the

$223 \mathrm{GO}$, the optimum recovery appeared at the setting of NoV aptamer of $5 \mu \mathrm{M}$ and GO of 0.05

$224 \mathrm{mg} / \mathrm{mL}$. A slightly strong quenching effect was found by GO compared to that of MWCNTs,

225 which can be attributed to the more interaction area of the sheet structure of GO than the tube

226 structure of MWCNTs. Thus a relative recovery rate can be achieved by GO. 
Fig. 3 and Fig. 4 also show the time-dependent fluorescence intensity changes. The time-

228 dependent response was studied to obtain a suitable incubation and reaction time for quenching

229 and recovery. As shown in the figures, the quenching of the 6-FAM NoV aptamer occurred

230 immediately after adding MWCNTs or GO, and a dramatic decline was observed within 5 min,

231 and then became a plateau after prolonging time. As indicated in the Fig. 3 and Fig. 4, the

232 recovery of the fluorescence could be completed within $5 \mathrm{~min}$, because no notable intensity

233 changes appeared afterwards. Thus 5 min was considered as the optimal incubation time for

234 quenching as well as the optimal recovery time.

235

236 Specificity of the biosensor

237 We evaluated the selectivity of our biosensor by detected the NoV VLPs solution mixed with

238 bovine serum albumin (BSA) and peptidoglycan, respectively. BSA was used as a blocking

239 buffer for the paper based device, while peptidoglycan is the major cell wall component of both

240 the gram positive and gram negative bacteria and may present in complex food matrices. The

241 mixture of BSA and NoV VLPs $(3.3 \mathrm{mg} / \mathrm{mL}$ and $129 \mathrm{ng} / \mathrm{mL}$ in final concentration), and the

242 mixture of peptidoglycan and NoV VLPs $(1.2 \mathrm{mg} / \mathrm{mL}$ and $129 \mathrm{ng} / \mathrm{mL}$ in final concentration)

243 were detected. The results as shown in Fig. 5 confirms the excellent specificity of the developed

244 biosensor. Notable changes in recovered fluorescence intensity were observed in all solutions

245 due to the presence of NoV VLPs. However, no distinguishable changes in recovered

246 fluorescence intensity were observed among the BSA or the peptidoglycan mixture with the NoV

247 VLPs solution indicating the absence of any cross-reactivity. The results also demonstrate that

248 the selected NoV aptamer can only specifically bind to the NoV VLPs. 


\section{Detection of NoV VLPs}

251 As a validation, a series of NoV VLPs dilution were detected by a microplate reader under the

252 optimized parameters. Fig. 6 shows the recovered fluorescence spectra versus various

253 concentration of NoV VLPs ranging from 0 to $12.9 \mu \mathrm{g} / \mathrm{mL}$. The recovered fluorescence intensity

254 increase with respect to the elevated protein concentration. No significant difference was

255 observed between the spectra of $1.29 \mathrm{ng} / \mathrm{mL}$ and that of after quenching. However,

256 distinguishable fluorescence intensity changes appear when the concentration increased to 12.9

$257 \mathrm{ng} / \mathrm{mL}$ for both the nanomaterials.

258 Under the optimized conditions, a series of NoV VLPs dilutions were assayed on the paper-

259 based microfluidic device. For multiplex detection, up to 5 sample dilutions and a negative

260 control could be assayed on a ready-to-use paper-based microfluidic device at one time. All tests

261 were performed at room temperature. To reduce the errors, the difference between the recovered

262 fluorescence intensity $\left(\mathrm{I}_{\mathrm{R}}\right)$ and after quenching $\left(\mathrm{I}_{0}\right)$ was used to determine the NoV VLPs

263 concentration in the sample. Fig. 7 shows the calibration curve of NoV VLPs assay by plotting

264 the changes in fluorescence intensity versus the different concentration, with the linear range of

$2650 \sim 12.9 \mu \mathrm{g} / \mathrm{mL}$ and $\mathrm{R}^{2}$ being 0.9696 for MWCNTs and 0.9818 for GO. The LoD is calculated by

$266 S_{b} S$ [23], where $S_{b}$ is the standard deviation of the blank measure, $S$ is the sensitivity

267 ( $\Delta$ concentration/ $\Delta$ intensity) and $k=3$ is numerical factor. The LoDs of the paper-based

268 microfluidic biosensor are $40 \mathrm{pM}$ for MWCNTs and $30 \mathrm{pM}$ for GO with 3\% -7\% in relative

269 standard deviation (RSD).

\section{Conclusions}


272 A paper-based microfluidic biosensor is developed for the detection of norovirus. The biosensor

273 utilized the FRET between the 6-carboxyfluorescein labelled norovirus aptamer and MWCNTs

274 and GO. The MWCNTs would quench the fluorescence of aptamer when they were mixed

275 together. In the presence of NoV, the fluorescence would be turned on (recovered) due to the

276 specific binding with the aptamer. The quantitative results showed a limit of detection of $40 \mathrm{pM}$

277 for MWCNTs and $30 \mathrm{pM}$ for GO, respectively. The easy-to-make paper-based microfluidic

278 biosensor could be easily applied for multi-virus diagnosis hence finds numerous applications in

279 food sector and clinical diagnosis.

281 Abbreviations

282 NoV: noroviruses; 6-FAM: 6-carboxyfluorescein; MWCNTs: multi-walled carbon nanotubes;

283 GO: graphene oxide; FRET: fluorescence resonance energy transfer; NoV VLPs: norovirus

284 virus-like particles; ELISA: enzyme-linked immunosorbent assay; RT-PCR: reverse transcription

285 polymerase chain reaction; LoD: limit of detection; LFAs: lateral-flow assays; POCT: point-of-

286 care testing.

\section{Authors' contributions}

288 XW and SN designed the study; XW performed experiments, acquired and analyzed data, XW

289 and SN drafted and edited the manuscript. All authors read and approved the final manuscript.

\section{Acknowledgements}

291 The authors sincerely thank the Natural Sciences and Engineering Research Council of Canada

292 (400705) for funding this study.

\section{Competing interests}

294 The authors declare that they have no competing interests. 


\section{Author details}

$296{ }^{1}$ BioNano Laboratory, School of Engineering, University of Guelph, Guelph, N1G 2W1

297 Canada. Email: xuanw@uoguelph.ca

$298 *$ BioNano Laboratory, School of Engineering, University of Guelph, Guelph, N1G 2W1

299 Canada. Email: sneethir@uoguelph.ca

301 References

302 1. Ahmed SM, Hall AJ, Robinson AE, Verhoef L, Premkumar P, Parashar UD, Koopmans

303 M, Lopman BA. Global prevalence of norovirus in cases of gastroenteritis: a systematic

304 review and meta-analysis. The Lancet infectious diseases. 2014; 14(8):725-30.

305 2. Koo HL, Ajami N, Atmar RL, DuPont HL. Noroviruses: the principal cause of foodborne 306 disease worldwide. Discovery medicine. 2010; 10 (50):61.

307 3. Glass RI, Parashar UD, Estes MK. Norovirus gastroenteritis. New England Journal of $308 \quad$ Medicine. 2009; 361(18):1776-85.

309 4. Jiang XI, Wang MI, Graham DY, Estes MK. Expression, self-assembly, and antigenicity 310 of the Norwalk virus capsid protein. Journal of virology. 1992; 66(11):6527-32.

311 5. Hayashi YU, Ando TA, Utagawa ET, Sekine SE, Okada SH, Yabuuchi K, Miki T, 312 Ohashi M. Western blot (immunoblot) assay of small, round-structured virus associated 313 with an acute gastroenteritis outbreak in Tokyo. Journal of clinical microbiology. 1989; 27(8):1728-33.

315 6. Vinjé J, Vennema H, Maunula L, von Bonsdorff CH, Hoehne M, Schreier E, Richards A, 316 Green J, Brown D, Beard SS, Monroe SS. International collaborative study to compare 
reverse transcriptase PCR assays for detection and genotyping of noroviruses. Journal of

$318 \quad$ clinical microbiology. 2003; 41(4):1423-33.

319 7. Sharma H, Mutharasan R. Review of biosensors for foodborne pathogens and toxins.

$320 \quad$ Sensors and actuators B: Chemical. 2013; 183:535-49.

321 8. Hong SA, Kwon J, Kim D, Yang S. A rapid, sensitive and selective electrochemical biosensor with concanavalin A for the preemptive detection of norovirus. Biosensors and Bioelectronics. 2015; 64:338-44. sensitive and selective electrochemical biosensor: Label-free detection of human norovirus using affinity peptide as molecular binder. Biosensors and Bioelectronics. 2017; 87:164-70. Development of lectin-linked immunomagnetic separation for the detection of hepatitis A virus. Viruses. 2014; 6(3):1037-48. Conrad JC. Detection of viruses by counting single fluorescent genetically biotinylated reporter immunophage using a lateral flow assay. ACS applied materials \& interfaces. 2015; 7(4):2891. Detection of Human Norovirus. mSphere. 2016; 1(5):e00219-16. 

Kourentzi K, Atmar RL, Willson RC. Sensitive detection of norovirus using phage nanoparticle reporters in lateral-flow assay. PloS one. 2015; 10(5):e0126571.

14. Davaji B, Lee CH. A paper-based calorimetric microfluidics platform for bio-chemical sensing. Biosensors and Bioelectronics. 2014; 59:120-6.

15. Zhang Y, Zuo P, Ye BC. A low-cost and simple paper-based microfluidic device for simultaneous multiplex determination of different types of chemical contaminants in food. Biosensors and Bioelectronics. 2015; 68:14-9.

346 16. Singh DK, Iyer PK, Giri PK. Role of molecular interactions and structural defects in the 347 efficient fluorescence quenching by carbon nanotubes. Carbon. 2012; 50(12):4495-505.

348 17. Alig I, Pötschke P, Lellinger D, Skipa T, Pegel S, Kasaliwal GR, Villmow T. 349 Establishment, morphology and properties of carbon nanotube networks in polymer melts. $350 \quad$ Polymer. 2012; 53(1):4-28.

351 18. Huang PJ, Liu J. DNA $\square$ length $\square$ dependent fluorescence signaling on graphene oxide $352 \quad$ surface. Small. 2012; 8(7):977-83.

353 19. Lin B, Yu Y, Li R, Cao Y, Guo M. Turn-on sensor for quantification and imaging of 354 acetamiprid residues based on quantum dots functionalized with aptamer. Sensors and 355 Actuators B: Chemical. 2016; 229:100-9.

356 20. Escudero-Abarca BI, Suh SH, Moore MD, Dwivedi HP, Jaykus LA. Selection, 357 characterization and application of nucleic acid aptamers for the capture and detection of 358 human norovirus strains. PLoS One. 2014; 9(9):e106805. 
21. Mu X, Zhang L, Chang S, Cui W, Zheng Z. Multiplex microfluidic paper-based immunoassay for the diagnosis of hepatitis C virus infection. Analytical chemistry. 2014; 86(11):5338-44.

22. Tian F, Lyu J, Shi J, Yang M. Graphene and graphene-like two-denominational materials based fluorescence resonance energy transfer (FRET) assays for biological applications. Biosensors and Bioelectronics. 2017; 89: 123-35.

23. Thomsen V, Schatzlein D, Mercuro D. Limits of detection in spectroscopy. Spectroscopy. $2003 ; 18(12): 112-4$.

371 Fig. 1 a Schematic illustration of turn-on sensor based on MWCNTs and 6-FAM functionalized

372 aptamer, illustrating the principle of the NoV detection based on the nanomaterial MWCNTs

373 (GO), Nov aptamer and NoV VLPs. The fluorescence of the NoV aptamer would be quenched 374 when they were mixed and coated on the paper surface due to the FRET process between the 375 probe and GO. In the presence of the NoV, the aptamer would bond to the protein because of the 376 stronger association constant between them, resulting in the fluorescence revocery. b Schematic

377 of the paper-based microfluidic device design for NoV detection using NoV aptamer 378 functionalized MWCNTs. $\mathbf{c}$ the picture of the paper-based microfluidic device.

379 Fig. 2 Characterization of the carboxylic acid functionalized MWCNTs and GO. a TEM images 380 of MWCNTs. b TEM images of GO. c UV-Vis absorption spectra of the MWCNTs and bare GO.

381 d Fluorescence spectra of 6-FAM NoV aptamer $(E x=490 \mathrm{~nm}, \mathrm{Em}=520 \mathrm{~nm})$ 
382 Fig. 3 Quenching effect of MWCNTs of various concentrations at a $1 \mu \mathrm{M}$ and $\mathbf{b} 5 \mu \mathrm{M}$ NoV 383 aptamer versus time. Recovery effect of MWCNTs of various concentrations at $\mathbf{c} 1 \mu \mathrm{M}$ and $\mathbf{d} 5$ $384 \mu \mathrm{M}$ NoV aptamer versus time.

385 Fig. 4 Quenching effect of GO of various concentrations at a $1 \mu \mathrm{M}$ and $\mathbf{b} 5 \mu \mathrm{M}$ NoV aptamer

386 versus time. Recovery effect of GO of various concentrations at c $1 \mu \mathrm{M}$ and $\mathbf{d} 5 \mu \mathrm{M} \mathrm{NoV}$ 387 aptamer versus time.

388 Fig. 5 Investigation of the selectivity of the biosensor by testing NoV VLPs sample with BSA 389 and peptidoglycan. a MWCNTs b GO.

390 Fig. 6 Fluorescence spectra of the 10-fold NoV VLPs serial dilution of NoV VLPs in buffer 391 ranging from 0 to $12.9 \mu \mathrm{g} / \mathrm{mL}$ by $\mathbf{a}$ MWCNTs $\mathbf{b}$ GO.

392 Fig. 7 Standard curves of the NoV VLPs detection by a MWCNTs. b GO on the paper-based 393 microfluidic device via the fluorescence intensity changes versus various NoV VLPs 394 concentration ranging from $0 \sim 12.9 \mu \mathrm{g} / \mathrm{mL}$. 
Na:

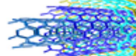

.
\#, Norovirus W-FAM-aptamer

MWCNTs Manched

Quenched
6-FAM-aptamer

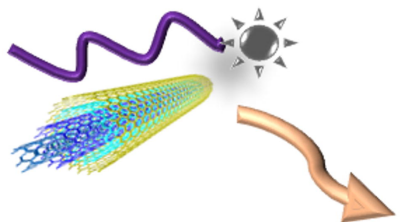

$\sum_{1 / 2}^{\prime \prime}$

C

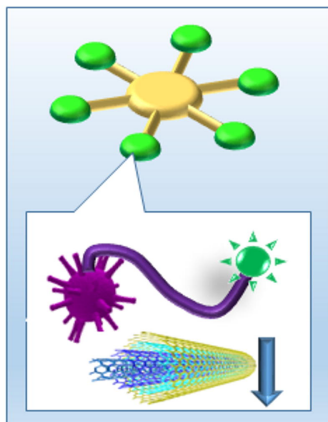

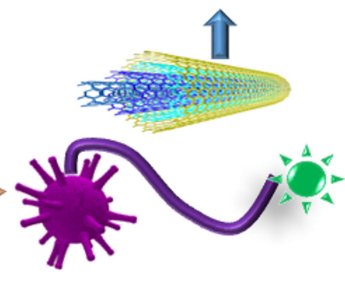

\section{Reaction well}

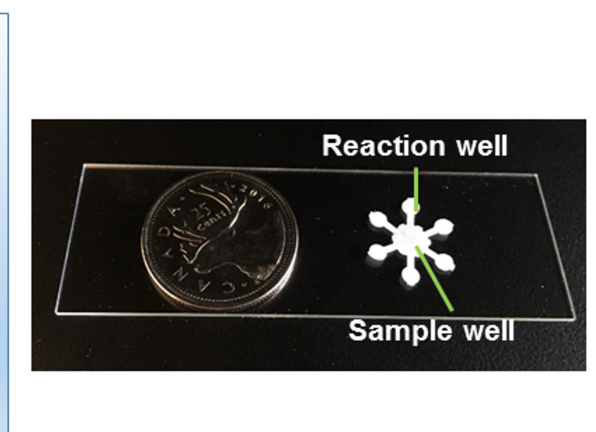

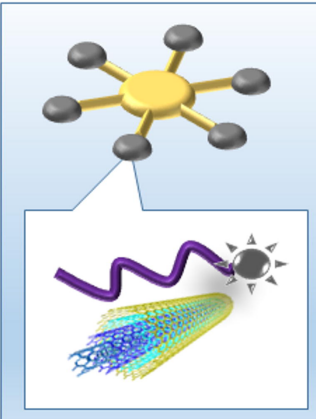


a

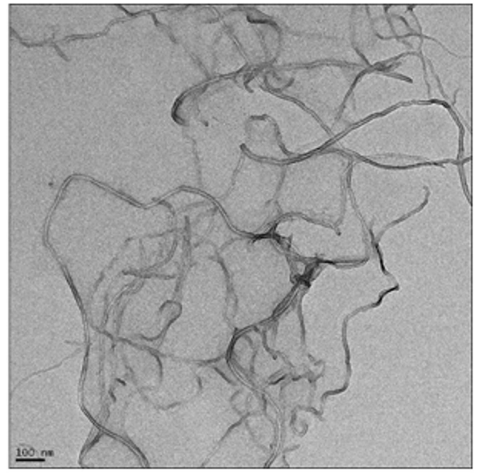

b

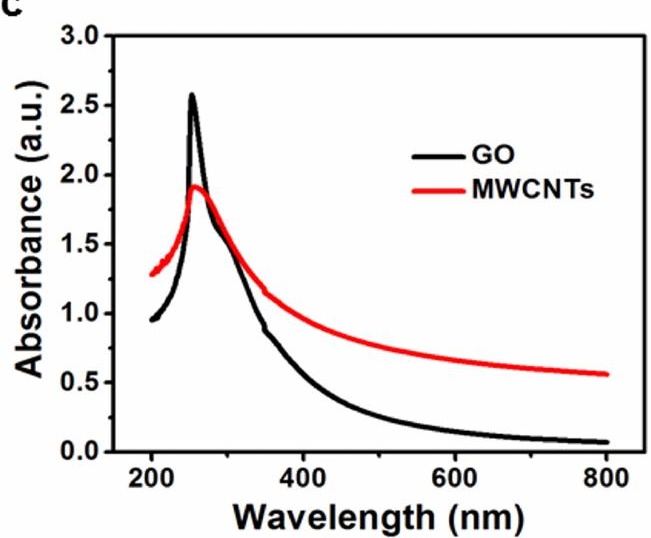

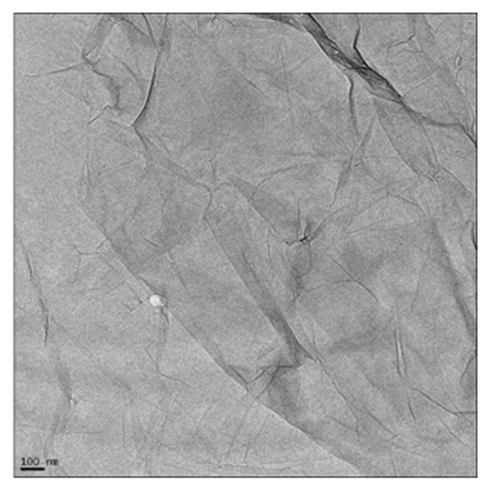

d

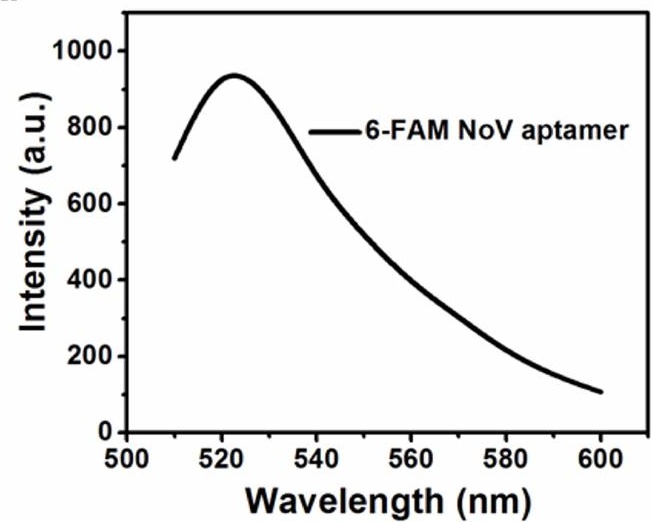



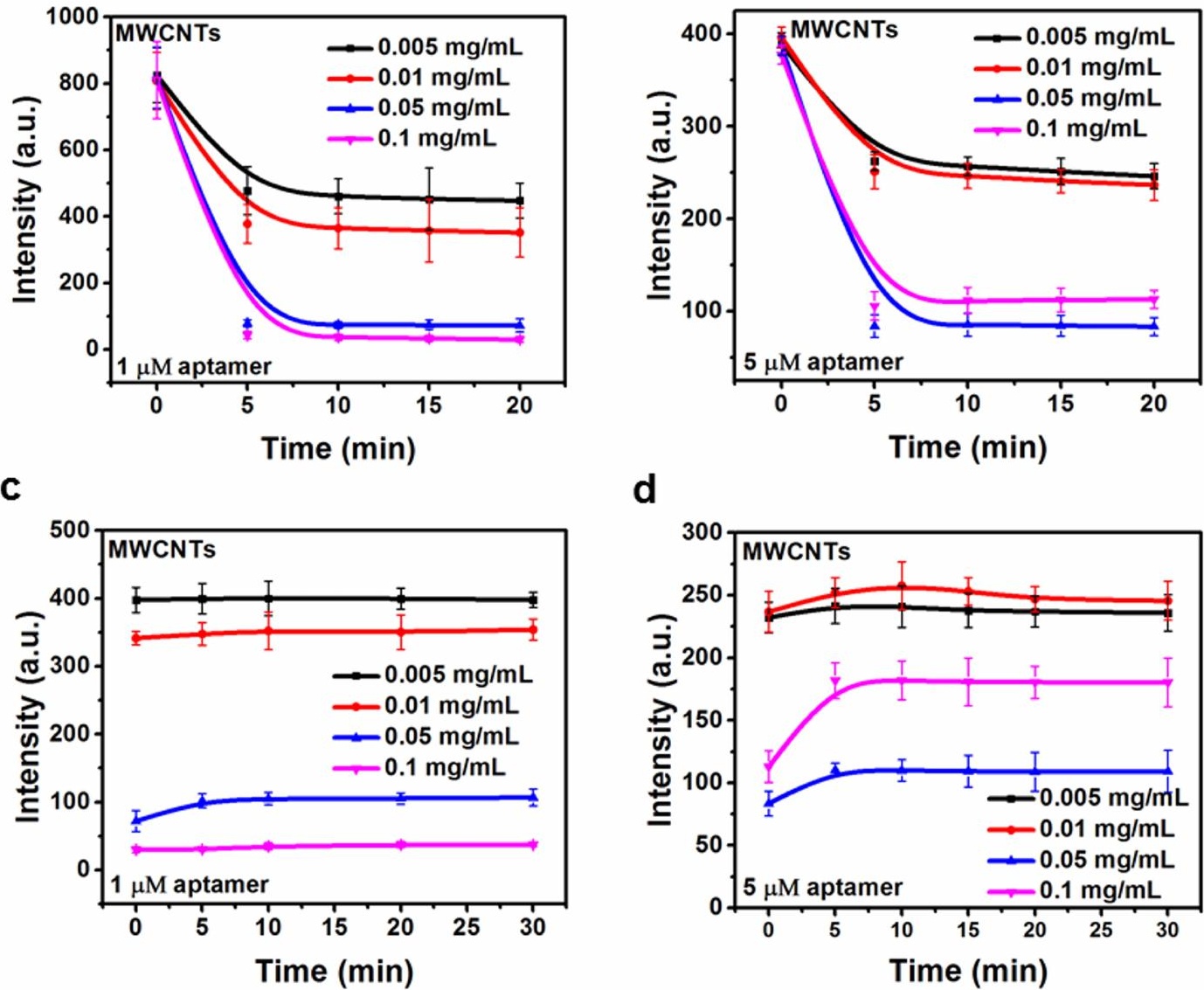

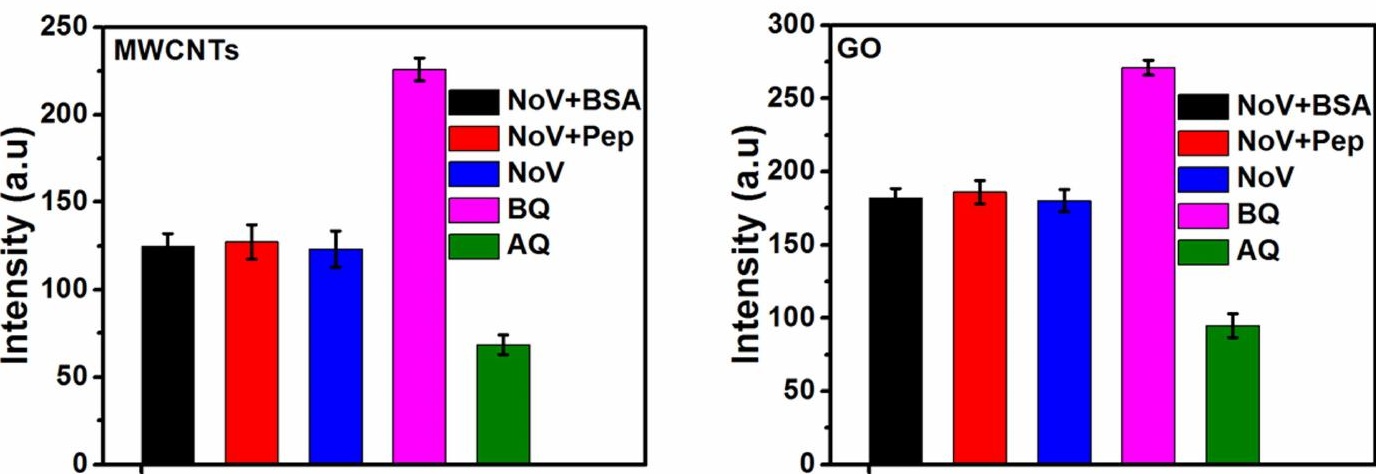

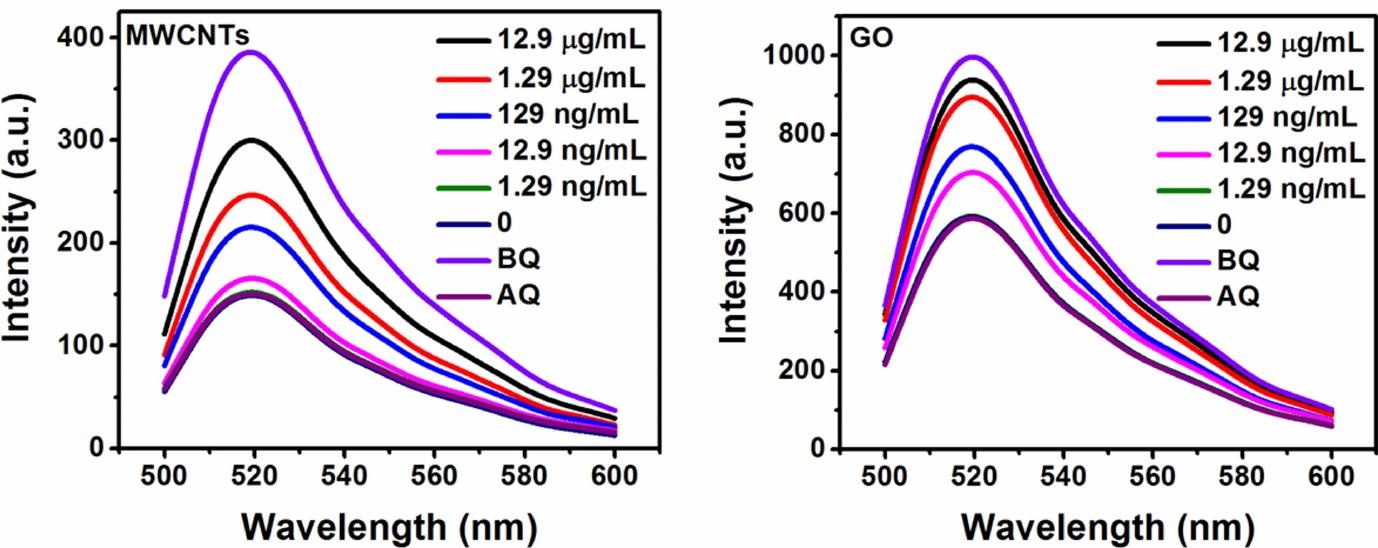
Ready-to-use paper-based biosensor

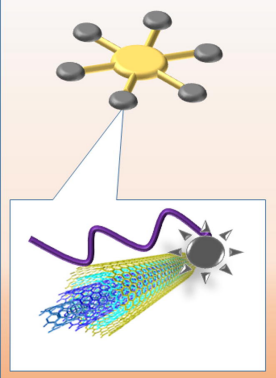

In the presence of norovirus

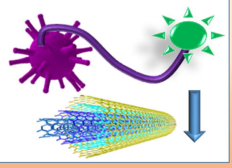

\section{Results}

Dynamic range:

$0 \sim 120 \mathrm{nM}$

Detection limits: MWCNTs-40 pM GO-30 pM 